\title{
NEAR-FIELD TRANSIENT WAVES IN ANISOTROPIC ELASTIC PLATES FOR TWO AND THREE DIMENSIONAL PROBLEMS*
}

\author{
R. A. ScotT ${ }^{\dagger}$ \\ Assistant Professor of Engineering Mechanics, The University of Michigan \\ and \\ J. MikLowitz \\ Professor of Applied Mechanics, California Institute of Technology
}

\begin{abstract}
The formal solutions to a class of non-axisymmetric problems involving a transversely isotropic elastic plate are derived. The Cagniard-deHoop method of inversion is used to obtain explicit solutions on the epicentral axis for certain materials, when a point shear load, with a "ramp" time-dependence, is applied to one face of the plate. Also treated is a problem involving plane-strain type motions, namely, the impulsive application of a normal line load to one face of an anisotropic plate, inversion for certain materials again being carried out on the epicentral axis. In both cases a numerical analysis of the solutions is presented.
\end{abstract}

\section{INTRODUCTION}

IN THE present paper the formal (transformed) solutions to a general class of non-axisymmetric, transient, elastic wave propagation problems involving a transversely isotropic, or hexagonal, infinite plate are derived - a generalization of some recent work of the authors [1] on isotropic plates. To illustrate the general theory, a particular case is examined, namely, the application of a point shear load, with a "ramp" time-dependence, to one face of the plate, and in the context of this problem, inversion is carried out explicitly for certain types of materials. Also treated is the excitation of plane-strain type waves in an anisotropic plate, by the impulsive application of a normal line load, again inversions being carried out explicitly for certain classes of materials. The response solutions for these problems are written for the epicentral axis, and in particular are analyzed numerically at the plate center for several specific anisotropic and isotropic solids.

The lack of knowledge regarding elastic transients in bounded anisotropic media is one of the main motivations behind the work at hand. As far as the authors are aware, the only existing studies on this topic are those of Hensel and Curtis [2], Suncheleev [3], and Rosenfeld and Miklowitz [4], on anisotropic rods, and a paper of the writers [5], in which the solutions to several problems involving plane-strain type waves in plates were given and analyzed numerically for the far-field. Both aspects of the present paper, i.e. nonaxisymmetric waves and the near-field in anisotropic waveguides, have of course received

* This work was sponsored by the Office of Naval Research under Contract Nonr-220(57), NR-064-487.

$\dagger$ Formerly, Research Fellow in Applied Mechanics at The California Institute of Technology. 
attention in the limit of isotropy. Besides the work of the writers [1], there should be mentioned the studies of Pytel and Davids [6], and Davids and Lawhead [7], who treated point shear and oblique impacts on one face of an infinite plate, and those of Harkrider [8], who, for a layered medium, considered the propagation of non-axisymmetric waves generated by time-harmonic, buried sources. Studies of the near-field in isotropic plates have been given by Mencher [9] who treated excitation by a spherically symmetric point source, and by Knopoff [10], Davids [11], Pytel and Davids [6] and [12], Davids and Lawhead [7], and Broberg [13], who considered surface load problems. Attention in the above papers was confined to the epicentral axis, but some exact information away from this axis has also been given by Rosenfeld and Miklowitz [14], who obtained the magnitudes, times of arrival, and locations of the wave fronts in infinite and semi-infinite plates, these fronts being generated by several types of surface and end loadings.

The formal solutions to the non-axisymmetric problems in the present work are obtained, as in [1], by a combination of multi-integral transform and integral superposition methods, whereas in the plane-strain example, multi-integral transforms alone are used. In both the point shear and line load problems, inversion for the epicentral axis was carried out by the Cagniard-de Hoop method. It is found that this technique applies, in its direct form, only for situations in which certain branch points are not on the real axis, and it is those circumstances which are treated in this paper.

\section{NON-AXISYMMETRIC WAVE PROPAGATION IN A TRANSVERSELY ISOTROPIC PLATE}

\subsection{The general problem}

Transverse isotropy and hexagonal symmetry are characterized by the existence of an axis- the $z$-axis below-with the property that in all planes perpendicular to it the material behaves isotropically. In terms of cylindrical coordinates $r, \theta, z$, the displacement equations of motion for such a medium are, in the absence of body forces (see, for instance, Mirsky [15]),

$$
\begin{aligned}
& {\left[a\left(\frac{\partial^{2}}{\partial r^{2}}+\frac{1}{r} \frac{\partial}{\partial r}-\frac{1}{r^{2}}\right)+\frac{e}{r^{2}} \frac{\partial^{2}}{\partial \theta^{2}}+c \frac{\partial^{2}}{\partial z^{2}}-\rho \frac{\partial^{2}}{\partial t^{2}}\right] u_{r}} \\
& \quad+\left[\frac{(a-e)}{r} \frac{\partial^{2}}{\partial \theta \partial r}-\frac{(a+e)}{r^{2}} \frac{\partial}{\partial \theta}\right] u_{\theta}+b \frac{\partial^{2} u_{z}}{\partial r \partial z}=0 \\
& {\left[\frac{(a-e)}{r} \frac{\partial^{2}}{\partial r \partial \theta}+\frac{(a+e)}{r^{2}} \frac{\partial}{\partial \theta}\right] u_{r}+\left[e\left(\frac{\partial^{2}}{\partial r^{2}}+\frac{1}{r} \frac{\partial}{\partial r}-\frac{1}{r^{2}}\right)+\frac{a}{r^{2}} \frac{\partial^{2}}{\partial \theta^{2}}\right.} \\
& \left.\quad+c \frac{\partial^{2}}{\partial z^{2}}-\rho \frac{\partial^{2}}{\partial t^{2}}\right] u_{\theta}+\frac{b}{r} \frac{\partial^{2} u_{z}}{\partial \theta \partial z}=0 \\
& b \frac{\partial}{\partial z}\left(\frac{\partial}{\partial r}+\frac{1}{r}\right) u_{r}+\frac{b}{r} \frac{\partial^{2} u_{\theta}}{\partial \theta \partial z}+\left[c\left(\frac{\partial^{2}}{\partial r^{2}}+\frac{1}{r} \frac{\partial}{\partial r}\right)+\frac{c}{r^{2}} \frac{\partial^{2}}{\partial \theta^{2}}\right. \\
& \left.\quad+d \frac{\partial^{2}}{\partial z^{2}}-\rho \frac{\partial^{2}}{\partial t^{2}}\right] u_{z}=0
\end{aligned}
$$


where the $u$ denote displacements, $t$ is the time, $\rho$ is the material density, and $a, b, c, d$, and $e$ are given in terms of the elastic constants $c_{i j}$ by : $a=c_{11}, b=c_{13}+c_{44}, c=c_{44}, d=c_{33}$, and $e=\frac{1}{2}\left(c_{11}-c_{12}\right)$. Reduction to isotropy is achieved by setting $a=d=\lambda+2 \mu, c=e=\mu$, and $b=\lambda+\mu$, where $\lambda$ and $\mu$ are Lamé's constants.

The pertinent stress-displacement relations are

$$
\begin{aligned}
\sigma_{z z} & =(b-c)\left(\frac{\partial u_{r}}{\partial r}+\frac{u_{r}}{r}\right)+\frac{b-c}{r} \frac{\partial u_{\theta}}{\partial \theta}+d \frac{\partial u_{z}}{\partial z} \\
\sigma_{z r} & =c\left(\frac{\partial u_{z}}{\partial r}+\frac{\partial u_{r}}{\partial z}\right) \\
\sigma_{z \theta} & =c\left(\frac{\partial u_{\theta}}{\partial z}+\frac{1}{r} \frac{\partial u_{z}}{\partial \theta}\right) .
\end{aligned}
$$

Taking the $z$-axis perpendicular to the plate surfaces, with the origin at the plate center, the boundary conditions are taken to be

$$
\begin{array}{ll}
\sigma_{z z}=g_{1}(\theta) T_{1}(t) I_{1}(r), & z=-H \\
\sigma_{z r}=g_{2}(\theta) T_{2}(t) I_{2}(r), & z=-H \\
\sigma_{z \theta}=-g_{3}(\theta) T_{2}(t) I_{2}(r), & z=-H \\
\sigma_{z z}=\sigma_{z r}=\sigma_{z \theta}=0, & z=H
\end{array}
$$

where $2 H$ is the plate thickness and the $g, T$, and $I$, are arbitrary functions.

Since the solution technique is similar to that used by the author's in [1], only an outline will be given here. The Laplace transform w.r.t. time is applied to (2.1) through (2.4) and then finite Fourier cosine and sine transforms are applied to (2.1), (2.3), (2.4), (2.5) and (2.2), (2.6), respectively. The $r$-dependence is suppressed by means of the superposition integrals

$$
\begin{aligned}
& \tilde{\bar{u}}_{r}^{c}=\int_{0}^{\infty}\left[\mathrm{X}_{1}(k, p, z) \frac{\mathrm{d}}{\mathrm{d} r} J_{\beta}(k r)+\mathrm{X}_{3}(k, p, z) \frac{\beta}{r} J_{\beta}(k r)\right] \mathrm{d} k \\
& \tilde{\bar{u}}_{2}^{c}=\int_{0}^{\infty} \mathrm{X}_{2}(k, p, z) J_{\beta}(k r) \mathrm{d} k \\
& \tilde{\bar{u}}_{\theta}^{S}=-\int_{0}^{\infty}\left[\mathrm{X}_{1}(k, p, z) \frac{\beta}{r} J_{j}(k r)+\mathrm{X}_{3} \frac{\mathrm{d}}{\mathrm{d} r} J_{\beta}(k r)\right] \mathrm{d} k
\end{aligned}
$$

where the bar denotes a Laplace transform, parameter $p$, the tilde denotes a Fourier transform, parameter $\beta$, the superscipts $C$ and $S$ denote a cosine or sine transform, respectively, $J_{\beta}$ denotes a Bessel function of the first kind of order $\beta$, and the $\mathrm{X}$ are functions to be determined. It is found that if equations (2.7), (2.8) and (2.9) are to satisfy the transformed cquations of motion, then the $\mathrm{X}$ must satisfy certain ordinary differential equations. Solutions to these equations are readily obtained and, using representation integrals based on inverse Hankel transforms, the arbitrary constants involved can be found on applying the transformed versions of the boundary conditions. Thus the transformed field quantities can be 
obtained and the procedure yields for the stresses:

$$
\begin{aligned}
\tilde{\bar{\sigma}}_{z z}^{c}= & -\frac{1}{2 c} \int_{0}^{\infty} \eta_{1}\left(M_{1} / F_{e}+M_{2} / F_{f}\right) J_{\beta}(k r) \mathrm{d} k \\
\tilde{\bar{\sigma}}_{r z}^{C}= & \frac{1}{2} \int_{0}^{\infty} \eta_{1}\left(M_{3} / F_{e}+M_{4} / F_{f}\right) \frac{\mathrm{d}}{\mathrm{d} r} J_{\beta}(k r) \mathrm{d} k \\
& +\tilde{g} \bar{T}_{2} \int_{0}^{\infty} \frac{\hat{I}_{2}^{\beta-1} \sinh \delta(H-z)}{\sinh 2 \delta H} \frac{\beta}{r} J_{\beta}(k r) \mathrm{d} k \\
\tilde{\tilde{\sigma}}_{z \theta}^{\mathrm{S}}= & -\frac{1}{2} \int_{0}^{\infty} \eta_{1}\left(M_{3} / F_{e}+M_{4} / F_{f}\right) \frac{\beta}{r} J_{\beta}(k r) \mathrm{d} k \\
& -\tilde{g} \bar{T}_{2} \int_{0}^{\infty} \frac{\hat{I}_{2}^{\beta-1} \sinh \delta(H-z)}{\sinh 2 \delta H} \frac{\mathrm{d}}{\mathrm{d} r} J_{\beta}(k r) \mathrm{d} k
\end{aligned}
$$

where

$$
\begin{aligned}
M_{1}= & h_{1} h_{2} \hat{I}_{2}^{\beta-1} \tilde{g} \bar{T}_{2}\left(\cosh \eta_{1} z \cosh \eta_{2} H-\cosh \eta_{2} z \cosh \eta_{1} H\right) \\
& +k c \tilde{g}_{1}^{C} \bar{T}_{1} \hat{I}_{1}^{\beta}\left(\frac{h_{2} f_{1}}{\eta_{1}} \cosh \eta_{2} z \sinh \eta_{1} H-\frac{h_{1} f_{2}}{\eta_{2}} \cosh \eta_{1} z \sinh \eta_{2} H\right) \\
M_{2}= & k c \tilde{g}_{1} \bar{T}_{1} \hat{I}_{1}^{\beta}\left(\frac{h_{1} f_{2}}{\eta_{2}} \sinh \eta_{1} z \cosh \eta_{2} H-\frac{h_{2} f_{1}}{\eta_{1}} \sinh \eta_{2} z \cosh \eta_{1} H\right) \\
& +h_{1} h_{2} \tilde{g} \bar{T}_{2} \hat{I}_{2}^{\beta-1}\left(\sinh \eta_{2} z \sinh \eta_{1} H-\sinh \eta_{1} z \sinh \eta_{2} H\right) \\
M_{3}= & \tilde{g} \bar{T}_{2} \hat{I}_{2}^{\beta-1}\left(\frac{f_{1} h_{2}}{\eta_{1}} \sinh \eta_{1} z \cosh \eta_{2} H-\frac{f_{2} h_{1}}{\eta_{2}} \sinh \eta_{2} z \cosh \eta_{1} H\right) \\
& +k c \frac{f_{1} f_{2}}{\eta_{1} \eta_{2}} \tilde{g}_{1}^{C} \bar{T}_{1} \hat{I}_{1}^{\beta}\left(\sinh \eta_{2} z \sinh \eta_{1} H-\sinh \eta_{1} z \sinh \eta_{2} H\right) \\
M_{4}= & k c \frac{f_{1} f_{2}}{\eta_{1} \eta_{2}} \tilde{g}_{1}^{C} \bar{T}_{1} \hat{I}_{1}^{\beta}\left(\cosh \eta_{1} z \cosh \eta_{2} H-\cosh \eta_{2} z \cosh \eta_{1} H\right) \\
& +\tilde{g} \bar{T}_{2} \hat{I}_{2}^{\beta-1}\left(\frac{f_{2} h_{1}}{\eta_{2}} \cosh \eta_{2} z \sinh \eta_{1} H-\frac{f_{1} h_{2}}{\eta_{1}} \cosh \eta_{1} z \sinh \eta_{2} H\right) \\
F_{e}= & \frac{\eta_{1}}{\eta_{2}} h_{1} f_{2} \cosh \eta_{1} H \sinh \eta_{2} H-h_{2} f_{1} \cosh \eta_{2} H \sinh \eta_{1} H \\
F_{f}= & \frac{\eta_{1}}{\eta_{2}} h_{1} f_{2} \sinh \eta_{1} H \cosh \eta_{2} H-h_{2} f_{1} \sinh \eta_{2} H \cosh \eta_{1} H \\
h_{j}= & {[b(b-c)-a d] k^{2}+c d \eta_{j}^{2}-\rho d p^{2}, \quad j=1,2 } \\
f_{j}= & (b-c) \eta_{j}^{2}+a k^{2}+\rho p^{2}, \quad j=1,2 \\
& j=l_{1} 2
\end{aligned}
$$




$$
\begin{aligned}
\eta_{j \sqrt{ }}(2 c d)= & {\left[\left(a d+c^{2}-b^{2}\right) k^{2}+(d+c) \rho p^{2}\right.} \\
& \left.-(-1)^{j}\left(A k^{4}+2 B \frac{\rho}{d} p^{2} k^{2}+\frac{\rho^{2} p^{4}}{d^{2}} D\right)^{1 / 2}\right]^{1 / 2}, \quad j=1,2 \\
A= & \left(a d+c^{2}-b^{2}\right)^{2}-4 a d c^{2} \\
B= & d\left[(d+c)\left(a d+c^{2}-b^{2}\right)-2 d c(a+c)\right] \\
D= & d^{2}(d-c)^{2} \\
\delta \sqrt{ }(c)= & \left(e k^{2}+\rho p^{2}\right)^{1 / 2} \\
\hat{I}_{1}^{\beta}= & \int_{0}^{\infty} r I_{1}(r) J_{\beta}(k r) \mathrm{d} r \\
\tilde{g}(\beta) \equiv & \tilde{g}_{2}^{c}=\tilde{g}_{3}^{S}(\beta)
\end{aligned}
$$

this last condition being necessary if the present mode of solution is to be applicable.

Equations (2.10), (2.11) and (2.12) constitute the formal solutions. Rather than attempt inversion in the context of the general problem, attention is now focused on a particular case.

\subsection{Point shear load problem}

The specific problem chosen for further study is the application of a point shear load, with a "ramp" time-dependence, to one face of the plate (Pytel and Davids [6], in their isotropic plate work, treated the same geometry, but the time-dependence was that of a Heaviside step). The boundary conditions are

$$
\begin{array}{ll}
\sigma_{z z}=0, \quad z=-H & \\
\sigma_{z r}=-\frac{\delta(r)}{\pi r} \cos \theta S(t), & z=-H \\
\sigma_{z \theta}=\frac{\delta(r)}{\pi r} \sin \theta S(t), & z=-H \\
\sigma_{z z}=\sigma_{z r}=\sigma_{z \theta}=0, & z=H
\end{array}
$$

where $\delta$ denotes the delta function and

$$
S(t)= \begin{cases}0, & t<0 \\ \frac{\sigma_{0} t}{Q}, & 0<t<Q \\ \sigma_{o}, & t>Q\end{cases}
$$

$\sigma_{o}$ being a constant and $Q$ a measure of the rise-time of the pulse. It should be noted that since $\theta$ cannot be defined at $r=0$, the point load must be interpreted as acting over a finite, but extremely small, area.

Inserting the above boundary conditions into (2.10) (2.11) and (2.12), and inverting the finite Fourier transforms, which presents no difficulties in the present case, the 
Laplace-transformed stresses are found to be

$$
\begin{aligned}
\frac{2 Q \pi c}{\sigma_{0} \cos \theta} \bar{\sigma}_{z z}= & -\frac{1}{p^{2}}\left(1-\mathrm{e}^{-p Q}\right) \int_{0}^{\infty}\left(M_{5} / F_{e}+M_{b} / F_{f}\right) J_{1}(k r) \mathrm{d} k \\
\frac{2 Q \pi}{\sigma_{o} \cos \theta} \bar{\sigma}_{r z}= & \frac{1}{p^{2}}\left(1-\mathrm{e}^{-p Q}\right) \int_{0}^{\infty}\left(M_{7} / F_{e}+M_{8} / F_{f}\right) \frac{\mathrm{d}}{\mathrm{d} r} J_{1}(k r) \mathrm{d} k \\
& -\frac{2}{p^{2}}\left(1-\mathrm{e}^{-p Q}\right) \int_{0}^{\infty} \frac{\sinh \delta(H-z)}{\sinh 2 \delta H} \frac{1}{r} J_{1}(k r) \mathrm{d} k
\end{aligned}
$$

where

$$
\begin{aligned}
& M_{5}=h_{1} h_{2} \eta_{1}\left(\cosh \eta_{1} H \cosh \eta_{2} z-\cosh \eta_{2} H \cosh \eta_{1} z\right) \\
& M_{6}=h_{1} h_{2} \eta_{1}\left(\sinh \eta_{2} H \sinh \eta_{1} z \quad \sinh \eta_{1} H \sinh \eta_{2} z\right) \\
& M_{7}=\frac{\eta_{1} f_{2} h_{1}}{\eta_{2}} \cosh \eta_{1} H \sinh \eta_{2} z-f_{1} h_{2} \cosh \eta_{2} H \sinh \eta_{1} z \\
& M_{8}=f_{1} h_{2} \sinh \eta_{2} H \cosh \eta_{1} z-\frac{\eta_{1} f_{2} h_{1}}{\eta_{2}} \sinh \eta_{1} H \cosh \eta_{2} z
\end{aligned}
$$

The expression for $-\left(2 Q \pi / \sigma_{o} \sin \theta\right) \bar{\sigma}_{z \theta}$ is the same as (2.14), except that the roles of $(\mathrm{d} / \mathrm{d} r) J_{1}(k r)$ and $(1 / r) J_{1}(k r)$ are interchanged.

The method of inversion best suited to near-field studies is the so-called CagniarddeHoop technique (see, for instance, Rosenfeld and Miklowitz [14]). To apply this technique, the various hyperbolic functions in equations such as (2.13) are expressed in terms of exponentials and then the denominators arising are expanded by means of the binomial theorem. Then the results are collected into a single series of exponentials, a form which has the advantage that each term of the series can be identified with a particular type of wave and so the early arriving disturbance can be assessed with the aid of just a few terms. Using large $p$ expansions it is found that the disturbance associated with $\eta_{1}, \eta_{2}$, and $\delta$, respectively, are (i) fully transverse wave traveling with speed $\sqrt{ } c / \rho$ in the $z$-direction, henceforth denoted by a subscript $S$, (ii) fully longitudinal wave traveling with speed $\sqrt{ } d / \rho$ in the $z$-direction, henceforth denoted by a subscript $P$ and (iii) fully transverse wave (of $S H$ type) traveling with speed $\sqrt{ } c / \rho$ in the $z$-direction, henceforth denoted by the subscripts $S H$.

Restricting attention to the epicentral axis, i.e. to $r=0$, which leads to the simplifications

$$
\frac{1}{r} J_{1}(k r)=\frac{\mathrm{d}}{\mathrm{d} r} J_{1}(k r)=k / 2
$$

it is found from (2.13) and (2.14) that $\bar{\sigma}_{z z}=0$ and that a few of the individual wave contributions to $\bar{\sigma}_{r z}$ are given by, in terms of the new variables $z=H(\alpha-1), k=p w \sqrt{ }(\rho / d)$,

$$
\begin{aligned}
& {\left[\frac{2 \pi Q \mathrm{~d} \bar{\sigma}_{a r}}{\rho \sigma_{o} \cos \theta}\right]_{P}=\left(1-\mathrm{e}^{-p Q}\right) \int_{0}^{\infty} \frac{w \eta_{1} \gamma_{2}}{\left(\eta_{2}-\eta_{1}\right) \Phi} \mathrm{e}^{-p H \alpha \eta_{2} v^{\prime}\left(\rho^{\prime} d\right)} \mathrm{d} w} \\
& {\left[\frac{2 \pi Q \mathrm{~d} \bar{\sigma}_{\alpha r}}{\rho \sigma_{o} \cos \theta}\right]_{S}=-\left(1-\mathrm{e}^{-p Q}\right) \int_{0}^{\infty} \frac{w \eta_{2} \gamma_{1}}{\left(\eta_{2}-\eta_{1}\right) \Phi} \mathrm{e}^{-p H \alpha \eta_{1}(\rho / \alpha)} \mathrm{d} w}
\end{aligned}
$$




$$
\begin{aligned}
& {\left[\frac{2 \pi Q \mathrm{~d} \bar{\sigma}_{\alpha r}}{\rho \sigma_{o} \cos \theta}\right]_{P S}=\left(1-\mathrm{e}^{-p Q}\right) \int_{0}^{\infty} \frac{w \eta_{2} \gamma_{1}}{\left(\eta_{2}-\eta_{1}\right) \Phi}(1+\Gamma) \mathrm{e}^{-p H\left((2-\alpha) \eta_{1}+2 \eta_{2}\right] \sqrt{ }(\rho / d)} \mathrm{d} w} \\
& {\left[\frac{2 \pi Q \mathrm{~d} \bar{\sigma}_{\alpha r}}{\rho \sigma_{o} \cos \theta}\right]_{S H}=-\left(1-\mathrm{e}^{-p Q}\right) \int_{0}^{\infty} w \mathrm{e}^{-p \alpha \delta \sqrt{ }(\rho / d)} \mathrm{d} w}
\end{aligned}
$$

where

$$
\begin{aligned}
\eta_{j}(w) \sqrt{ }(2 c d) & =\left[\left(a d+c^{2}-b^{2}\right) w^{2}+d(d+c)-(-1)^{j}\left(A w^{4}+2 B w^{2}+D\right)^{\frac{1}{2}}\right]^{\frac{1}{2}}, \quad j=1,2, \\
\gamma_{j}(w) & =\left[(b-c)^{2} w^{2}-d L(w)\right] \eta_{j}^{2}(w)+d L(w), \quad j=1,2, \\
\Phi(w) & =d L(w)+\left[d L(w)-(b-c)^{2} w^{2}\right\rceil\left[\frac{1}{c d} L(w) N(w)\right]^{\frac{1}{2}} \\
N(w) & =c w^{2}+d, L(w)=a w^{2}+d \\
\Gamma(w) & =\psi_{+}(w) / \psi_{-}(w) \\
\psi_{ \pm}(w) & =h_{1}(w) \eta_{1}(w) f_{2}(w) \pm h_{2}(w) \eta_{2}(w) f_{1}(w) \\
h_{j}(w) & =[b(b-c)-a d] w^{2}+c d \eta_{j}^{2}(w)-d^{2}, \quad j=1,2, \\
f_{j}(w) & =(b-c) \eta_{j}^{2}(w)+a w^{2}+d, \quad j=1,2, \\
\delta(w) \sqrt{ }(c) & =\left(e w^{2}+d\right)^{\frac{1}{2}} .
\end{aligned}
$$

In equation (2.17) the subscripts $P S$ denote the contribution from a $P$ wave reflected at the lower surface of the plate as an $S$ wave. It should be noted that the expressions are not valid at $\alpha=2$ (the lower surface of the plate), since there, for instance, the fact that the $P S$ wave adds to the $P$ wave would have to be taken into account. It should also be noted that the sequence in which the terms are listed is not meant to imply their order of occurrence, which can change from material to material and from station to station. It should be noted that the expressions for $-2 \pi Q \bar{\sigma}_{z \theta} /\left(\sigma_{o} \sin \theta\right)$ are the same as the right hand sides of (2.15), (2.16), (2.17), and (2.18), and so attention hereafter is restricted to $\bar{\sigma}_{z r}$.

The integrals in (2.15), (2.16), (2.17), and (2.18), are now in a form to which the final step of the present mode of inversion can be applied. The essence of this step is to try and make substitutions such that the above integrals become Laplace transforms, since if this can be achieved, inversion follows by inspection, provided no non-integrable singularities arise. For example, this goal can be reached in (2.15) on making the substitutions

$$
t_{P}=H \alpha \eta_{2} \sqrt{ }(\rho / d), \quad t_{P Q}=Q+t_{P}
$$

provided these new variables can be identified with time, that is to say, provided $\eta_{2}$ is real, positive, and monotonically increasing, on the real, positive $w$-axis. If this is not so, then paths in the $w$-plane must be sought along which it is true and which are equivalent (in the sense of the Cauchy-Goursat theorem) to the original path of integration. In this indirect fashion (2.15) would again reduce to a Laplace transform and inversion would follow immediately, provided no non-integrable singularities arise. Clearly the preceding discussion also applies to (2.16), (2.17), and (2.18), except that the appropriate substitutions are

$$
\begin{aligned}
t_{S} & =\alpha \eta_{1} H \sqrt{ }(\rho / d), \quad t_{S Q}=Q+t_{S} \\
t_{P S} & =\left[(2-\alpha) \eta_{1}+2 \eta_{2}\right] H \sqrt{ }(\rho / d), \quad t_{P S Q}=Q+t_{P S} \\
t_{S H} & =H \alpha \delta \sqrt{ }(\rho / d), \quad t_{S H Q}=Q+t_{S H} .
\end{aligned}
$$


The question of the location of paths-the so-called Cagniard-deHoop paths--in the $w$-plane such that on them substitutions such as (2.20) can be interpreted as time variables depends on the multi-valued structure of the $\eta_{j}(w)$. Though the general issue is quite complicated, as can be seen from the work of Kraut [16] on unbounded anisotropic media, if attention is confined to the epicentral axis then circumstances arise for which the analysis is considerably simplified. A detailed investigation of this area has been given elsewhere by the authors [17], and only a brief summary will be presented here. Equation (2.19) shows that in general the $\eta_{j}(w)$ have six branch points and it was found that two major categories arose, namely, (i) two branch points on the imaginary axis and four in the complex plane, (ii) two branch points on the imaginary axis, and four on the real axis. For case (i) it was shown that, on suitably defining the branches, the $\eta_{j}$ are positive, real, and monotonic increasing on the positive, rea! $w$-axis, and hence for substitutions such as $(2.20)$ the Cagniard-deHoop path is the positive, real $w$-axis, i.e. the original contour of integration. For case (ii) the direct method fails in that the $\eta_{j}$ become complex on the original contour of integration. Using a set of criteria developed by Musgrave [18], it was found in a numerical study of several materials that when branch points fall on the real axis then the wave surfaces were such that cuspidal edges were being intercepted. Though Kraut [16] and Duff [19] treated the interception of cuspidal edges in connection with some unbounded media work, here the situation is more complicated in that one has to contend with the multiple reflection of such singular wave surfaces. Another interesting item in the area is the possibility of intercepting other singularities, such as conical points (recently examined by Burridge [20] in a study connected with infinite media). It seems clear that the various branch point situations are closely connected with physical singularities and it is hoped to pursue such questions further in a later work.

Focusing on materials and orientations for which no branch points occur on the real axis, Cagniard-deHoop substitutions such as (2.20) are directly applicable and (2.15), for instance, becomes

$$
\begin{aligned}
{\left[\frac{2 \pi Q \mathrm{~d} \bar{\sigma}_{\alpha \mathrm{r}}}{\rho \sigma_{o} \cos \theta}\right]_{P}=} & \frac{1}{\alpha H} / \frac{d}{\rho} \int_{H \alpha_{v}(\rho / d)}^{\infty}\left[\frac{w \eta_{1} \gamma_{2}}{\left(\eta_{2}-\eta_{1}\right) \Phi\left(\mathrm{d} \eta_{2} / \mathrm{d} w\right)}\right]_{w=w\left(t_{P}\right)} \mathrm{e}^{-p t_{P}} \mathrm{~d} t_{P} \\
& -\frac{1}{\alpha H} / \frac{d}{\rho} \int_{Q+H \alpha_{v}(\rho / d)}^{\infty}\left[\frac{w \eta_{1} \gamma_{2}}{\left(\eta_{2}-\eta_{1}\right) \Phi\left(\mathrm{d} \eta_{2} / \mathrm{d} w\right)}\right]_{w^{\prime}=w_{\left(t_{P Q}\right)}} \mathrm{e}^{-p t_{P Q}} \mathrm{~d} t_{P Q}
\end{aligned}
$$

where $w=w\left(t_{P}\right)$ and $w=w\left(t_{P Q}\right)$ are the appropriate roots of $(2.20)$. On the introduction of suitable Heaviside step functions, the integrals in (2.24) hecome I aplace transforms, and so inversion is immediate, giving

$$
\begin{aligned}
{\left[\frac{2 \pi Q \mathrm{~d} \sigma_{\alpha r}}{\rho \sigma_{o} \cos \theta}\right]_{P}=} & \frac{1}{\alpha}\left[\frac{w \eta_{1} \gamma_{2}}{\left(\eta_{2}-\eta_{1}\right) \Phi\left(\mathrm{d} \eta_{2} / \mathrm{d} w\right)}\right]_{w=w\left(\tau_{P}\right)} H\left(\tau_{P}-\alpha\right) \\
& -\frac{1}{\alpha}\left[\frac{w \eta_{1} \gamma_{2}}{\left(\eta_{2}-\eta_{1}\right) \Phi\left(\mathrm{d} \eta_{2} / \mathrm{d} w\right)}\right]_{w=w\left(\tau_{P Q}\right)} H\left[\tau_{P Q}-\left(\alpha+\tau_{Q}\right)\right]
\end{aligned}
$$

where the following dimensionless times have been introduced

$$
\tau_{P}=\frac{t_{P}}{H} \sqrt{\frac{d}{\rho}}, \quad \tau_{P Q}=\frac{t_{P Q}}{H} / \frac{d}{\rho}, \quad \tau_{Q}=\frac{Q}{H} \quad \frac{d}{\rho}
$$

and $H(\tau)$ denotes the Heaviside step function. 
In a similar fashion, (2.16), (2.17), and (2.18) can be shown to give

$$
\begin{aligned}
{\left[\frac{2 \pi Q \mathrm{~d} \sigma_{\alpha r}}{\rho \sigma_{o} \cos \theta}\right]_{S}=} & -\frac{1}{\alpha}\left[\frac{w \eta_{2} \gamma_{1}}{\left(\eta_{2}-\eta_{1}\right) \Phi\left(\mathrm{d} \eta_{1} / \mathrm{d} w\right)}\right]_{w=w(\tau s)} H\left(\tau_{s}-\alpha / \frac{d}{c}\right) \\
& +\frac{1}{\alpha}\left[\frac{w \eta_{2} \gamma_{1}}{\left(\eta_{2}-\eta_{1}\right) \Phi\left(\mathrm{d} \eta_{1} / \mathrm{d} w\right)}\right]_{w=w\left(\tau_{s Q}\right)} H\left(\tau_{S Q}-\alpha / \frac{d}{c}-\tau_{Q}\right) \\
{\left[\frac{2 \pi Q \mathrm{~d} \sigma_{\alpha r}}{\rho \sigma_{o} \cos \theta}\right]_{P S}=} & \left.\frac{w \eta_{2} \gamma_{1}(1+\Gamma)}{\left(\eta_{2}-\eta_{1}\right) \Phi\left[(2-\alpha)\left(\mathrm{d} \eta_{1} / \mathrm{d} w\right)+2\left(\mathrm{~d} \eta_{2} / \mathrm{d} w\right)\right]}\right\}_{w=w\left(\tau_{P S}\right)} \\
& \times H\left[\tau_{P S}-(2-\alpha) / \frac{d}{c}-2\right] \\
& -\left\{\frac{w \eta_{2} \gamma_{1}(1+\Gamma)}{\left(\eta_{2}-\eta_{1}\right) \Phi\left[(2-\alpha)\left(\mathrm{d} \eta_{1} / \mathrm{d} w\right)+2\left(\mathrm{~d} \eta_{2} / \mathrm{d} w\right)\right]}\right\}_{w=w\left(\tau_{P S Q}\right)} \\
& \times H\left[\tau_{P S Q}-(2-\alpha) / \frac{d}{c}-2-\tau_{Q}\right] \\
{\left[\frac{2 \pi Q \mathrm{~d} \sigma_{\alpha r}}{\rho \sigma_{0} \cos \theta}\right]_{S H}=} & -\frac{1}{\alpha}\left[\frac{w}{(\mathrm{~d} \delta / \mathrm{d} w)}\right]_{w=w\left(\tau_{S H)}\right.} H\left(\tau_{S H}-\alpha / \frac{d}{c}\right) \\
& +\frac{1}{\alpha}\left[\frac{w}{(\mathrm{~d} \delta / \mathrm{d} w)}\right]_{w=w\left(\tau_{S H Q}\right)} H\left(\tau_{S H Q}-\alpha / \frac{d}{c}-\tau_{Q}\right)
\end{aligned}
$$

where

$$
\begin{aligned}
\tau_{S} & =\eta_{1}(w) \alpha \\
\tau_{S Q} & =\tau_{Q}+\tau_{S} \\
\tau_{P S} & =(2-\alpha) \eta_{1}+2 \eta_{2} \\
\tau_{P S Q} & =\tau_{Q}+\tau_{P S} \\
\tau_{S H} & =\delta(w) \alpha \\
\tau_{S H Q} & =\tau_{Q}+\tau_{S H}
\end{aligned}
$$

and $w=w\left(\tau_{S}\right), w=w\left(\tau_{S Q}\right), w=w\left(\tau_{P S}\right), w=w\left(\tau_{P S Q}\right), w=w\left(\tau_{S H}\right)$, and $w=w\left(\tau_{S H Q}\right)$, are the appropriate roots of (2.29), (2.30), (2.31), (2.32), (2.33) and (2.34), respectively.

A numerical analysis of these and similar expressions is given in section four of the paper.

\section{THE IMPULSIVE APPLICATION OF A NORMAL LINE LOAD TO ONE FACE OF AN ANISOTROPIC INFINITE PLATE}

In this section a further contribution to the study of the near-field in anisotropic waveguides is given in the context of the title problem (which was studied by Broberg [13], in the limit of isotropy).

Several orientations of the crystallographic axes are to be considered and in order to preserve generality, dimensionless coordinates $\xi, \eta, \zeta$ are used. Each of these coordinates is 
to be interpreted as one of the crystallographic axes* $x, y, z$, divided by the plate halfthickness $H$. In terms of these coordinates, the line load is taken parallel to the $\eta$-axis and the plate surfaces are specified by $\zeta= \pm 1$. Under these circumstances (and assuming zero initial conditions and body forces), it is found for certain classes of anisotropy that the line load gives rise to waves of the plane-strain type, the equations of motion of which are

$$
\begin{aligned}
& a \frac{\partial^{2} u_{\xi}}{\partial \xi^{2}}+b \frac{\partial^{2} u_{\xi}}{\partial \xi \partial \zeta}+\frac{\partial^{2} u_{\xi}}{\partial \xi^{2}}=d \frac{\partial^{2} u_{\xi}}{\partial \tau^{2}} \\
& c \frac{\partial^{2} u_{\xi}}{\partial \xi^{2}}+b \frac{\partial^{2} u_{\xi}}{\partial \xi \partial \zeta}+d \frac{\partial^{2} u_{\xi}}{\partial \zeta^{2}}=d \frac{\partial^{2} u_{\xi}}{\partial \tau^{2}}
\end{aligned}
$$

where $a, b, c$ and $d$ are related to the elastic constants in the manner shown in Table 1, and $\tau$ is the dimensionless time $H \tau=t \sqrt{ }(d / \rho)$. The equations for the orientations: $H \xi=z$, $H \zeta=x ; H \xi=y, H \zeta=x ; H \xi=z, H \zeta=y$, which also admit of plane-strain motions, can be obtained from (3.1) and (3.2) on setting $a=d, d=a$. Physically, the cases involving three constants correspond to cubic crystals, those involving five constants correspond to hexagonal crystals and transversely isotropic materials, those involving six constants correspond to certain types of tetragonal crystals, and those involving nine constants correspond to orthorhombic crystals and orthotropic materials.

Under plane-strain conditions, the pertinent stress-displacement relations are

$$
\begin{aligned}
& H \sigma_{\xi \xi}=d \frac{\partial u_{\xi}}{\partial \zeta}+(b-c) \frac{\partial u_{\xi}}{\partial \xi} \\
& H \sigma_{\xi \xi}=c\left(\frac{\partial u_{\xi}}{\partial \zeta}+\frac{\partial u_{\xi}}{\partial \xi}\right) \\
& H \sigma_{\xi \xi}=a \frac{\partial u_{\xi}}{\partial \xi}+(b-c) \frac{\partial u_{\xi}}{\partial \zeta} .
\end{aligned}
$$

The stresses for the orientations: $H \xi=z, H \zeta=x ; H \xi=y, H \zeta=x ; H \xi=z, H \zeta=y$, can be obtained from (3.3), (3.4), and (3.5) by setting $a=d, d=a$.

The boundary conditions for the problem are

$$
\begin{aligned}
& \left.\begin{array}{l}
\sigma_{\zeta \zeta}=-\sigma_{o} \delta(\xi) \delta(\tau) \\
\sigma_{\zeta \zeta}=0
\end{array}\right\} \zeta=-1 \\
& \sigma_{\zeta \xi}=\sigma_{\zeta \xi}=0, \quad \zeta=1
\end{aligned}
$$

where $\sigma_{o}$ is a load constant.

The details of the solution technique are quite similar to those given by, for instance, Rosenfeld and Miklowitz [14], and only an outline of the process will be given here. Taking Laplace and Fourier exponential transforms, (3.1) and (3.2) reduce to a set of coupled ordinary differential equations, whereas (3.3), (3.4) and (3.5) express transformed stresses in terms of transformed displacements. The solutions to the ordinary differential equations involve four arbitrary constants, which can be evaluated on applying the transformed boundary conditions, which are obtained from (3.6) and (3.7). In this fashion the transformed field quantities are obtained and again inversion is approached by means of the CagniarddeHoop method. Restricting attention to materials and orientations for which no branch

* The notation as regards these axes is that given in Love [21]. 
points occur on the real axis and confining interest to the epicentral axis $(\xi=0)$, a few of the individual wave contributions to the normal displacement $u_{\alpha}\left(u_{\xi}=0\right.$ for $\left.\xi=0\right)$, where $\alpha=\zeta+1$, can be shown to be

$$
\begin{aligned}
{\left[\frac{c \pi u_{\alpha}}{H \sigma_{o}}\right]_{P}=} & \frac{c}{\alpha}\left[\frac{L \Theta_{1}}{\left(\eta_{2}-\eta_{1}\right) \Phi\left(\mathrm{d} \eta_{2} / \mathrm{d} w\right)}\right]_{w=w\left(\tau_{P}\right)} H\left(\tau_{P}-\alpha\right) \\
{\left[\frac{c \pi u_{\alpha}}{H \sigma_{o}}\right]_{S}=} & -\frac{c}{\alpha}\left[\frac{L \Theta_{2}}{\left(\eta_{2}-\eta_{1}\right) \Phi\left(\mathrm{d} \eta_{1} / \mathrm{d} w\right)}\right]_{w=w\left(\tau_{s}\right)} H\left(\tau_{S}-\alpha / \frac{d}{c}\right) \\
{\left[\frac{c \pi u_{\alpha}}{H \sigma_{o}}\right]_{P S}=} & -c\left\{\frac{L \Theta_{2}(1-\Gamma)}{\left(\eta_{2}-\eta_{1}\right) \Phi\left[(2-\alpha)\left(\mathrm{d} \eta_{1} / \mathrm{d} w\right)+2\left(\mathrm{~d} \eta_{2} / \mathrm{d} w\right)\right]}\right\}_{w=w\left(\tau_{P S}\right)} \\
& \times H\left(\tau_{P S}-(2-\alpha) / \frac{d}{c}-2\right)
\end{aligned}
$$

where

$$
\Theta_{j}=1-\eta_{j}^{2}-\frac{b-c}{d} w^{2}, \quad j=1,2,
$$

and all other functions are the same as in the point shear load problem, except that $a, b, c$, and $d$ have the broader interpretation given in Table 1 . It should be noted that the above

\begin{tabular}{|c|c|c|c|c|}
\hline \multicolumn{5}{|c|}{ Axes: $H \xi=x, H \zeta=z$} \\
\hline $\begin{array}{l}\text { No. of elastic } \\
\text { constants }\end{array}$ & $a$ & $b$ & $c$ & $d$ \\
\hline 3 & $c_{11}$ & $c_{12}+c_{44}$ & $c_{44}$ & $c_{11}$ \\
\hline 5 & $c_{11}$ & $c_{13}+c_{44}$ & $c_{44}$ & $c_{33}$ \\
\hline 6 & $c_{11}$ & $c_{13}+c_{44}$ & $c_{44}$ & $c_{33}$ \\
\hline 9 & $c_{11}$ & $c_{13}+c_{s s}$ & $c_{55}$ & $c_{33}$ \\
\hline \multicolumn{5}{|c|}{ Axes : $H \xi=x, H \zeta=y$} \\
\hline $\begin{array}{l}\text { No. of elastic } \\
\text { constants }\end{array}$ & $a$ & $b$ & $c$ & $d$ \\
\hline 3 & $c_{11}$ & $c_{12}+c_{44}$ & $c_{44}$ & $c_{11}$ \\
\hline 5 & $c_{11}$ & $\frac{1}{2}\left(c_{11}+c_{12}\right)$ & $\frac{1}{2}\left(c_{11}-c_{12}\right)$ & $c_{11}$ \\
\hline 6 & $c_{11}$ & $c_{12}+c_{66}$ & $c_{66}$ & $c_{11}$ \\
\hline 9 & $c_{11}$ & $c_{12}+c_{66}$ & $c_{66}$ & $c_{22}$ \\
\hline \multicolumn{5}{|c|}{ Axes: $H \xi=y, H \zeta=z$} \\
\hline $\begin{array}{l}\text { No. of elastic } \\
\text { constants }\end{array}$ & $a$ & $b$ & $c$ & $d$ \\
\hline 5 & $c_{11}$ & $c_{13}+c_{44}$ & $c_{44}$ & $c_{33}$ \\
\hline 6 & $c_{11}$ & $c_{13}+c_{44}$ & $c_{44}$ & $c_{33}$ \\
\hline 9 & $c_{22}$ & $c_{23}+c_{44}$ & $c_{44}$ & $c_{33}$ \\
\hline
\end{tabular}
expressions are not valid at the lower surface of the plate since there, for instance, the $P$ and PS waves combine.

TABLE 1. ElaStic PARAMETERS For THE ClasSES OF MATERIALS AND ORIENTATIONS UNDER CONSIDERATION 
A numerical discussion of (3.8), (3.9), (3.10), and other expressions where necessary is given in the following section.

\section{NUMERICAL RESULTS}

In this section some numerical studies of (2.25), (2.26), (2.27), (2.28), (3.8), (3.9), (3.10), and other wave contributions where necessary, are presented for the plate center $(\alpha=1)$. The materials treated in connection with the point shear load problem are the transversely isotropic medium ice, the hexagonal medium cobalt, and isotropic media with Poisson's ratio $\sigma=0.10$ and 0.45 for comparison purposes. The solids studied in the line load problem, for some specific orientations of the axes of symmetry, are the cubic media sodium chloride and pyrites, the hexagonal medium cobalt, the transversely isotropic medium ice, the orthorhombic medium $\alpha$-uranium, the orthotropic medium beechwood, and isotropic media with $\sigma=0.10,0.20,0.30$ and 0.45 . All the above materials are such that no branch points occur on the real axis.

The mode of computation adopted was as follows : For a given material, orientation of the axes of symmetry and value of the rise time $\tau_{Q}$ the sequence of arrivals was determined. Then the pertinent contributions, i.e. expressions such as (2.26), and the various functions of $\tau$ involved, i.e. expressions such as (2.29), are computed as functions of $w$. The elastic constants used, which were obtained from Hearmon ([22] and [23]), are shown below in Table 2, all units being $10^{11} \mathrm{dynes} / \mathrm{cm}^{2}$.

TABLE 2. VAlues of THE Elastic CONSTANTS

\begin{tabular}{lccccccccc}
\hline \multicolumn{1}{c}{ Materials } & $c_{11}$ & $c_{22}$ & $c_{33}$ & $c_{44}$ & $c_{55}$ & $c_{66}$ & $c_{12}$ & $c_{13}$ & $c_{23}$ \\
\hline Sodium chloride & 4.83 & - & - & 1.26 & - & - & 1.25 & - & - \\
Pyrites & 36.1 & - & - & 10.5 & - & - & -4.7 & - & $\cdots$ \\
Beryl & 26.94 & - & 23.63 & 6.53 & - & - & 9.61 & 6.61 & - \\
Ice & 1.36 & - & 1.46 & 0.32 & - & - & 0.67 & 0.52 & - \\
Cobalt & 30.70 & - & 35.81 & 7.55 & - & - & 16.50 & $10 \cdot 27$ & - \\
$\alpha$-uranium & 21.47 & 19.86 & 26.71 & 12.44 & 7.34 & 7.43 & 4.65 & $2 \cdot 18$ & $10 \cdot 76$ \\
Beechwood & 0.170 & 1.580 & 0.338 & 0.156 & 0.044 & 0.103 & 0.150 & 0.135 & 0.222 \\
\hline
\end{tabular}

Shown in Figs. 1 and 2 are the dimensionless mid-plate stresses as a function of time in the point shear load problem, for ice, cobalt and isotropic media with $\sigma=0.10$ and 0.45 . These diagrams show that the response as regards overall behaviour is quite similar in both anisotropic and isotropic media. Thus in all cases the stresses undergo finite jumps at the shear $(S, S H)$ and delayed shear $(S Q, S H Q)$ arrivals and the maximum stresses occur at the $S Q, S H Q$ arrival. There are differences in the "fine structure" of the responses in the isotropic and anisotropic solids, as evidenced by the more pronounced effect of the $P Q$ phase in the anisotropic media. One other feature that should be mentio..ed is the difference in the sequence of arrivals for $\sigma=0.45$ over those in the other media.

The dimensionless mid-plate, vertical displacements in the line load problem are presented in Figs. 3-7 as a function of time. Certain specific orientations of the axes of symmetry are considered and the media are sodium chloride, pyrites, cobalt, ice, $\alpha$-uranium, beechwood, and isotropic media with $\sigma=0 \cdot 10,0 \cdot 20,0.30$ and 0.45 . Before embarking on any detailed discussion, some overall features should first be noted. On observing that 


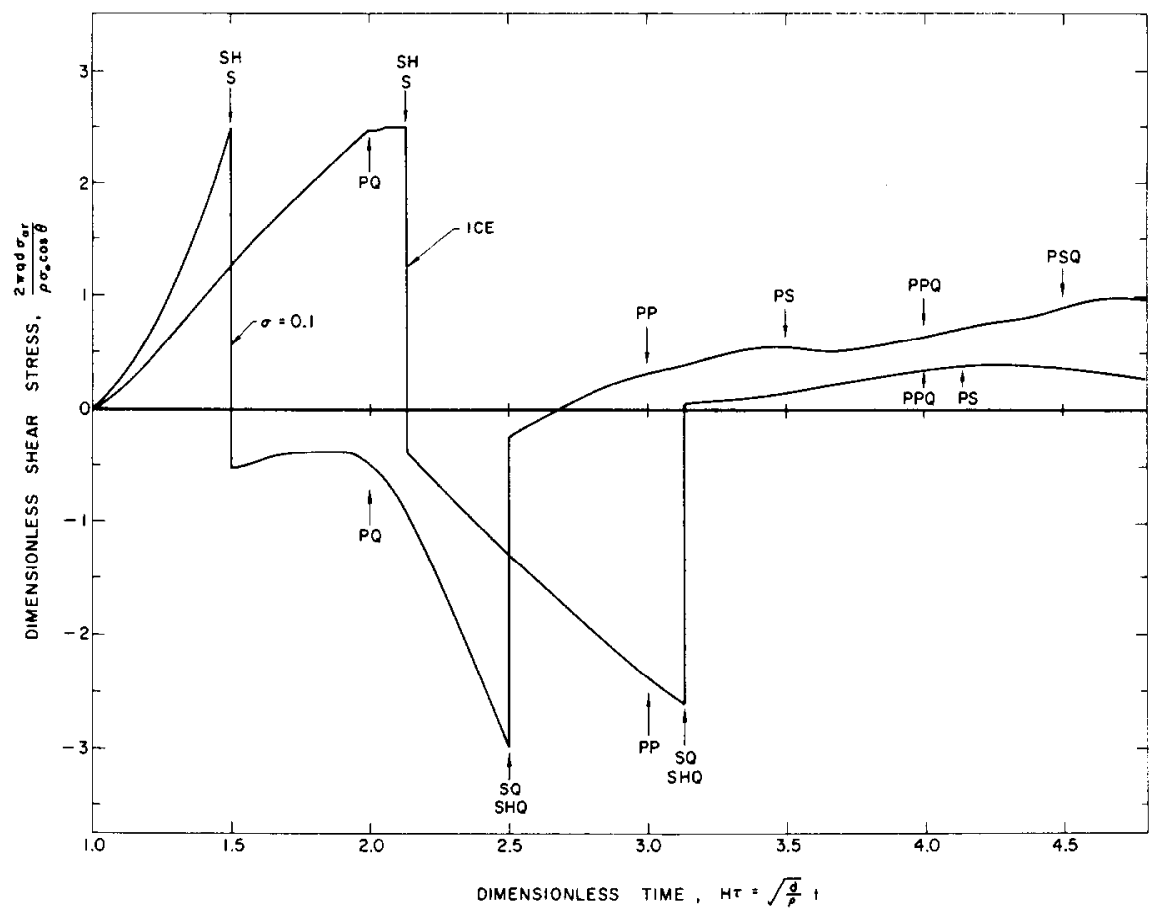

FIG. 1. Midplate stress, for point shear load problem, as a function of time for the transversely isotropic medium ice and an isotropic medium with $\sigma=0 \cdot 10$. Rise-time $\tau_{Q}$ of input pulse $=1 \cdot 0$.

$\eta_{1}=\sqrt{ } d / c, \eta_{2}=1$, when $w=0$, it follows from (3.8), (3.9), and (3.11) that the $P$ portion of the disturbance starts with an infinite amplitude, whereas the $S$ portion starts with zero amplitude. Moreover, it can be seen that the $P P, P P P$, etc. phases also begin with an infinite amplitude, whereas all other phases, such as $P S, S S, P P S$ etc., start with zero amplitude. The infinite amplitudes (which behave like $\tau^{-\frac{1}{2}}$ as $\tau \rightarrow 0$ ) are of course due to the singular nature of the load time-dependence, but it was felt that the effects of anisotropy could be exhibited on a broader scale within the present framework, due to prohibitive labor associated with a large number of computations for other time-dependencies.

Figures 3 and 4 give responses for the cubic media pyrites and sodium chloride (the disturbance is the same for the three cubic axes), and isotropic media with $\sigma=0 \cdot 10,0 \cdot 20$, 0.30 and 0.45. Again, the isotropic materials differ in their behaviour from the anisotropic ones, as evidenced by the contributions from the $S$ phase. For pyrites and sodium chloride, the $S$ wave contribution is quite small, whereas it is very pronounced in the various isotropic media, the smaller $\sigma$, the bigger being the effect. Also, the response in both anisotropic media shows a uniform decrease after the $P P$ phase (until the arrival of the $P P P$ pulse), whereas in the isotropic media there is a period of decrease followed by an increase.

The disturbances for the transversely isotropic material ice, the hexagonal medium cobalt, and the orthorhombic material $\alpha$-uranium are presented in Fig. 5 , the axes in all cases being: $H \xi=y, H \zeta=z$. The overall features are similar to those present in the cubic media, but again are different from those in any isotropic solid, in the weakness of the $S$ phase, and the ultimate decay after the $P P$ arrival (until the PPP arrival). Inspection of 


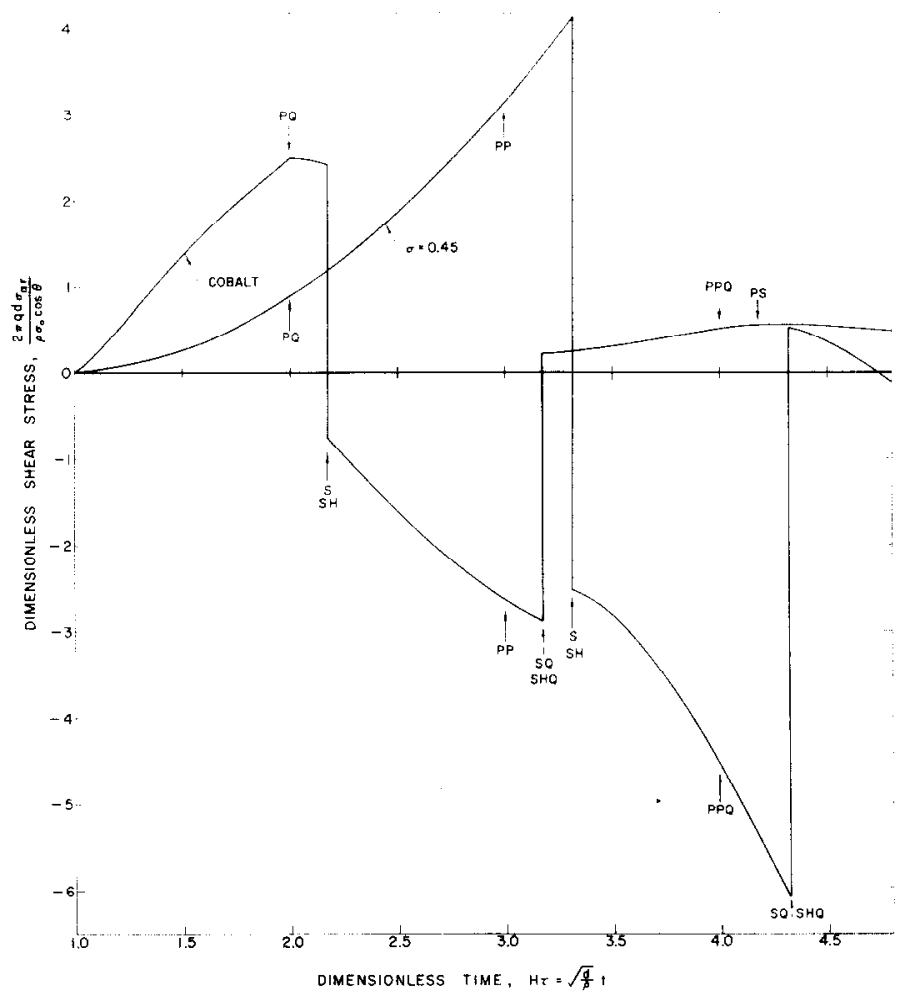

FIG. 2. Midplate stress, for point shear load problem, as a function of time for the hexagonal medium cobalt and an isotropic medium with $\sigma=0.45$. Rise-time $\tau_{Q}$ of input pulse $=1 \cdot 0$.

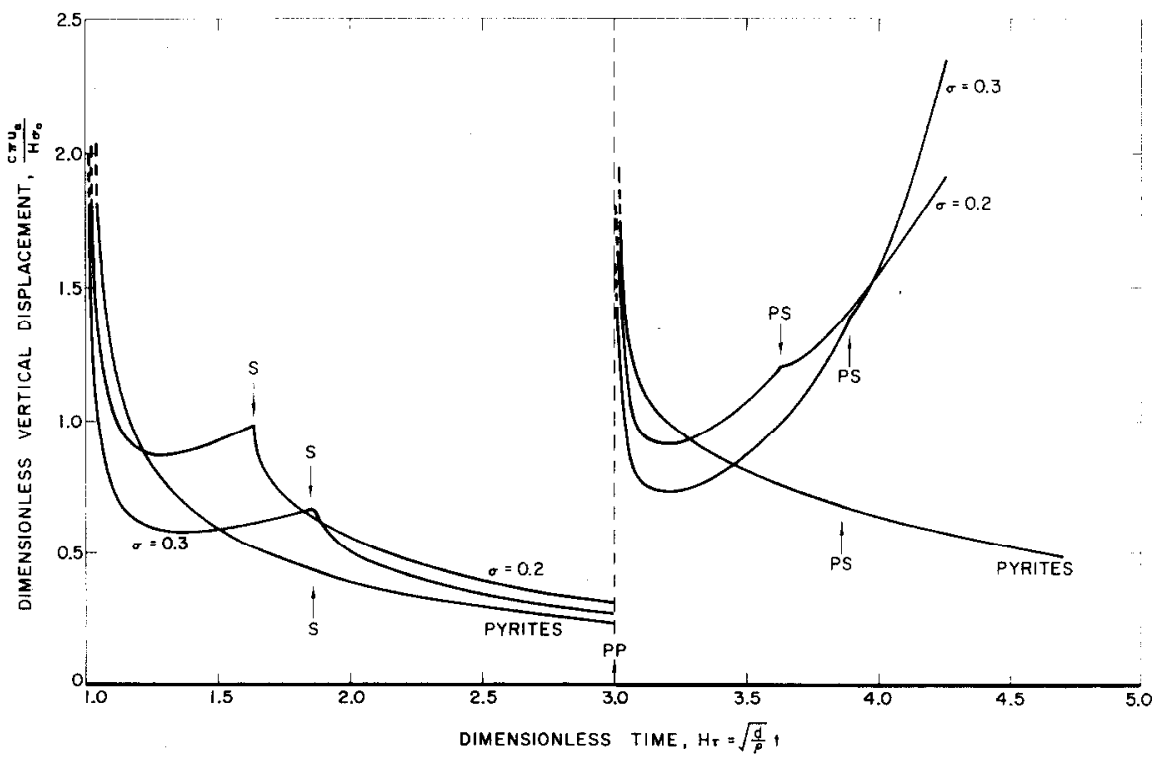

FIG. 3. Midplate vertical displacement, for line load problem, as a function of time for the cubic medium pyrites and for isotropic media with $\sigma=0.20$ and 0.30 . 


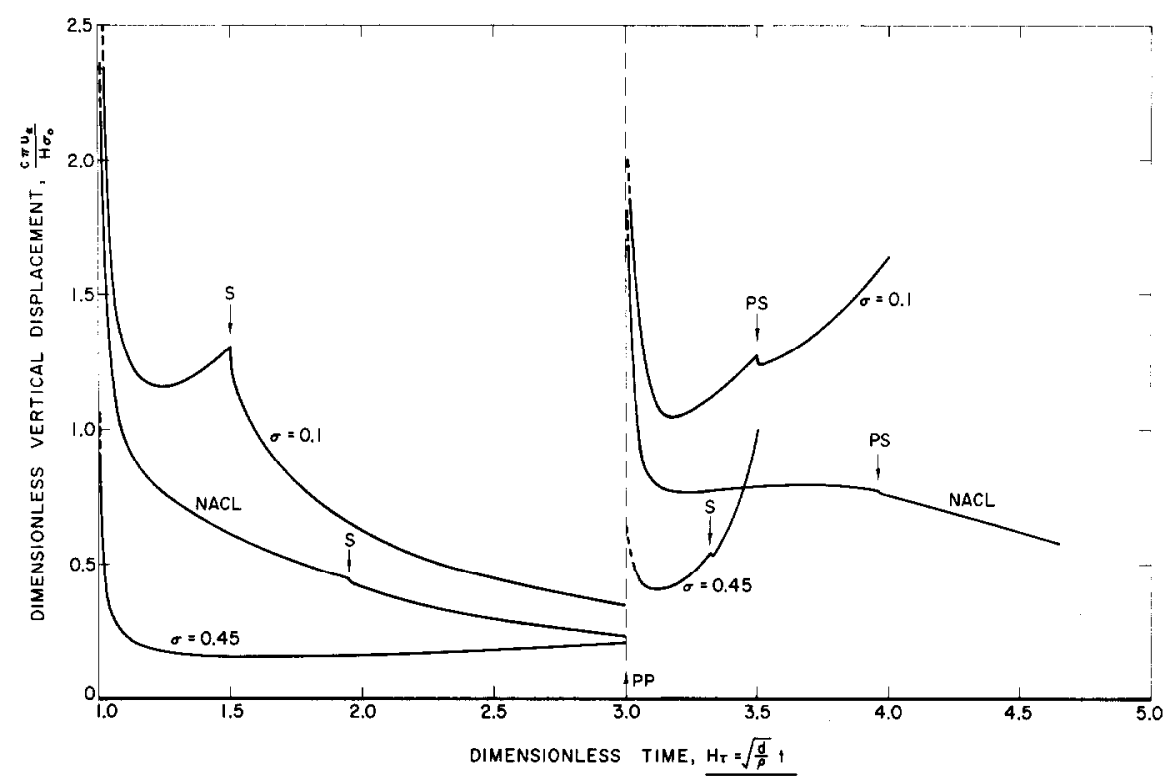

FIG. 4. Midplate vertical displacement, for line load problem, as a function of time for the cubic medium sodium chloride and for isotropic media with $\sigma=0.10$ and 0.45 .

Figs. 3, 4 and 5 shows that some differences exist between the various anisotropic media, particularly in the interval between the $P P$ and $P S$ waves, in which the amplitudes in pyrites and $\alpha$-uranium decrease uniformly, whereas those in ice, cobalt and sodium chloride exhibit a maximum.

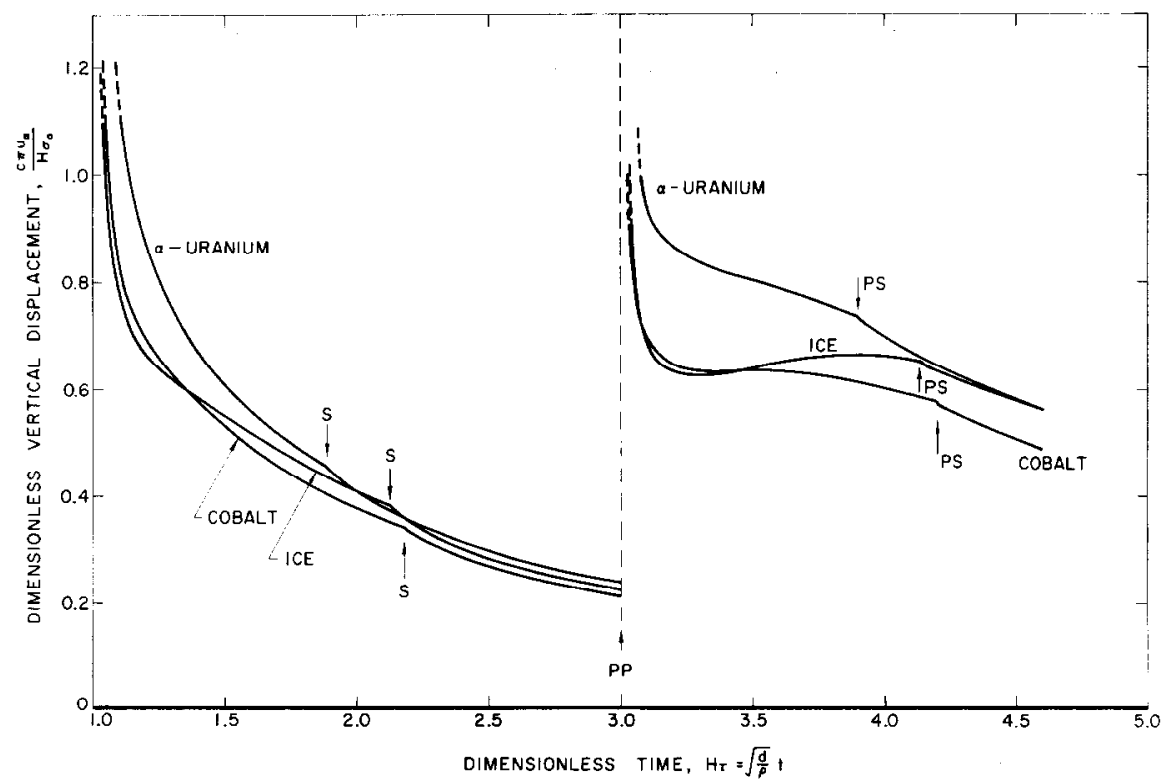

FIG. 5. Midplate vertical displacement, for line load problem, as a function of time for the hexagonal medium cobalt, the transversely isotropic medium ice (axes in both cases: $H \xi=y, H \zeta=z$ ), and the orthorhombic medium $\alpha$-uranium (axes: $H \xi=y, H \zeta=z$ ). 


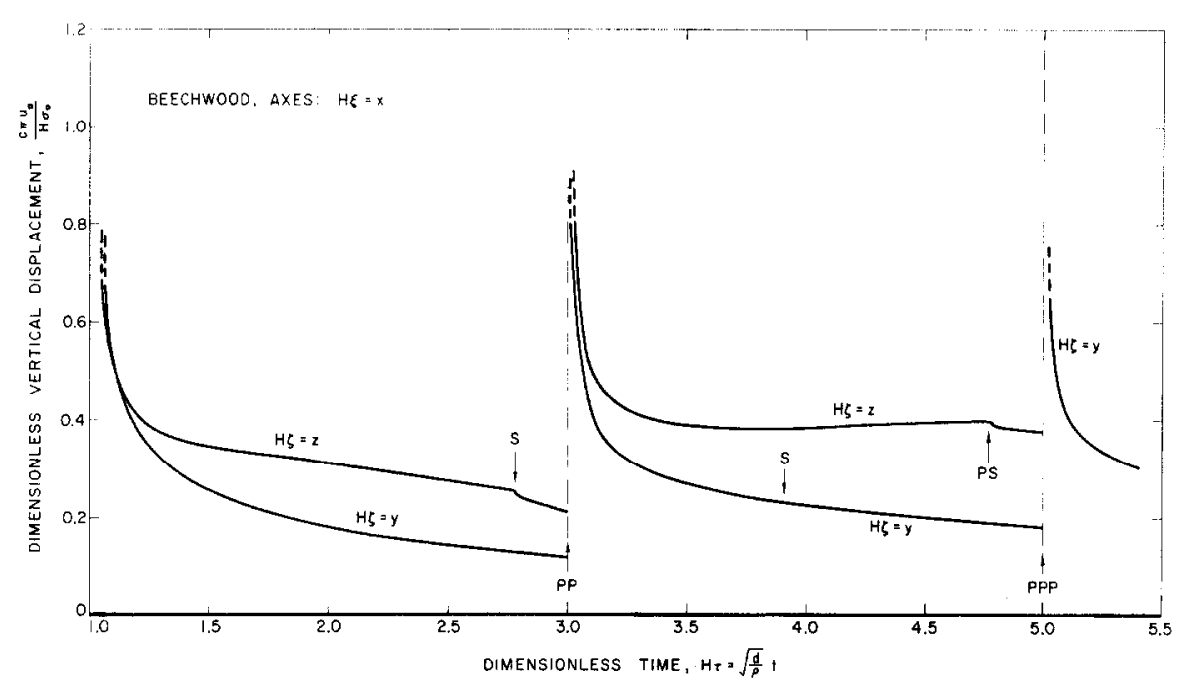

FIG. 6. Midplate vertical displacement, for line load problem, as a function of time for the orthotropic material beechwood (axes: $H \xi=x, H \zeta=y$, and $H \xi=x, H \zeta=z$ ).

One measure of anisotropy could be the effect on the response of the orientation of the axes of symmetry with respect to the plate surfaces. This is illustrated in Figs. 6 and 7 in which the response for the orthotropic medium beechwood is given for the orientations (i) $H \xi=x, H \zeta=y$, (ii) $H \xi=x, H \zeta=z$, and (iii) $H \xi=y, H \zeta=z$. As the diagrams show, this effect can be quite pronounced. One notable difference is in the sequence of arrivals. For (i) it is $P, P P, S, P P P$, whereas for (ii) and (iii) it is $P, S, P P, P S$. Other noteworthy items are the risc in amplitude in (iii) after the $P$ phase - in common with isotropic media-a feature which is absent in (i) and (ii), and the strong increase in (iii) after the $P P$ arrival, which is again similar to an isotropic response, except for the rapid decay after the arrival of the $P S$ wave.

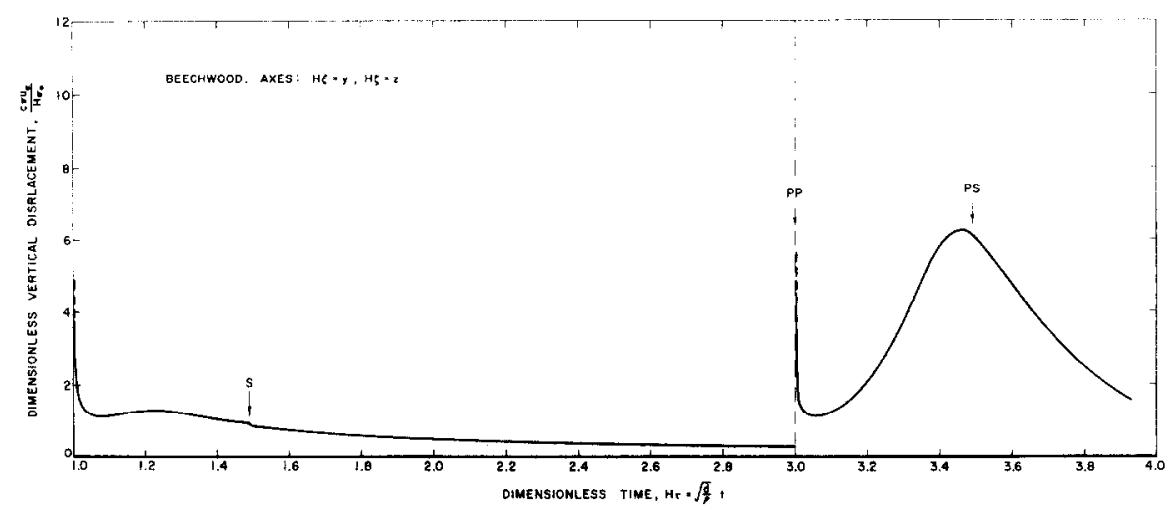

FIG. 7. Midplate vertical displacement, for line load problem, as a function of time for the orthotropic material beechwood (axes: $H \xi=y, H \zeta=z$ ). 


\section{REFERENCES}

[1] R. A. ScotT and J. MikLowitz, Transient non-axisymmetric wave propagation in an infinite isotropic elastic plate. Int. J. Solids Struct. 5, 65-79 (1969).

[2] R. D. Hensel and C. W. Curtis, Propagation of a longitudinal strain pulse along a single-crystal bar J. appl. Phys. 38, 2679 (1967).

[3] R. LA. SuncheleEv, Dynamic problems for a transversely isotropic elastic cylinder. $P M M$ 30, 1156-1160 (1967).

[4] R. Rosenfeld and J. Miklowitz, Elastic wave propagation in rods of arbitrary cross section. J. appl. Mech. 32, 290-294 (1965).

[5] R. A. ScotT and J. Miklowitz, Transient elastic waves in anisotropic plates. J. appl. Mech. 34, 104-110 (1967).

[6] A. Pytel and N. Davids, Transient analysis of shear impact. J. appl. Mech. 29, 33-39 (1962).

[7] N. Davids and W. Lawhead, Transient analysis of oblique impact on plates. J. Mech. Phys. Solids 13, $199-212$ (1965).

[8] D. G. HARKRIDER, Surface waves in multilayered elastic media-I. Rayleigh and Love waves from buried sources in a multilayered elastic half-space. Bull. Seism. Soc. Am. 54, 627-679 (1964).

[9] A. G. Mencher, Epicentral displacement caused by elastic waves in an infinite slab. J. appl. Phys. 24, 12401246 (1953).

[10] L. KNOPOFF, Surface motions of a thick plate. J. appl. Phys. 29, 661-670 (1958).

[11] N. Davids, Transient analysis of stress-wave penetration in plates. J. appl. Mech. 26, 651-660 (1959).

[12] A. Pytel and N. DAVIDS, Further transient analysis of stress wave propagation in plates. Proc. 4th Midwest. Conf. Solid Mech. 358-381 (1959).

[13] K. B. Broberg, A problem on stress waves in an infinite elastic plate, K. tek. Högsk Handl. Rept. No. 139 (1959).

[14] R. Rosenfeld and J. Miklowitz, Wave fronts in elastic rods and plates. Proc. 4th U.S. Nat. Congr. appl. Mech., 293-303 (1962).

[15] I. Mirsky, Wave propagation in transversely isotropic circular cylinders-I: Theory. J. acoust. Soc. Am. 37, 1016 (1965).

[16] E. A. Kraut, Advances in the theory of anisotropic elastic wave propagation. Rev. Geophys. 1, 401-448 (1963).

[17] R. A. Scotr and J. Miklowitz, Transient elastic waves in infinite anisotropic plates: The near-field. ONR Tech. Rept. No. 4, Contract Nonr-220 (57), NR-064-487 (1966).

[18] M. J. P. Musgrave, On whether elastic wave surfaces possess cuspidal edges. Proc. Camb. phil. Soc. 53, 897-906 (1957).

[19] G. F. D. Duff, The cauchy problem for elastic waves in an anisotropic medium. Phil. Trans. R. Soc. Lond. A252, 249-273 (1960).

[20] R. BURRIDGE, The singularity on the plane lids of the wave surface of elastic media with cubic symmetry. Q. J. Mech. appl. Math. 20, 41-56 (1967).

[21] A. E. H. Love, A Treatise on the Mathematical Theory of Elasticity, 4th ed. Dover (1944).

[22] R. F. S. Hearmon, The elastic constants of anisotropic materials. Adv. Phys. 5, 323-382 (1956).

[23] R. F. S. Hearmon, Applied Anisotropic Elasticity. Oxford University Press (1961).

(Received 25 November 1968 ; revised 14 March 1969)

\footnotetext{
Абстракт-Дается формальное решение для класса не-осесимметрических задач, касаюшихся поперечно изотропной упругой пластинки. Используется метод инверсии Канярда-де Гупа для получения решений в явной форме, на основе оси эпицентра, для некоторых материалов, если точка контакта сдвига, с зависимостью от времени типа "откоса", приложенная к одной стороне пластинки. Обсуждается также задача, касающаяся движения типа плоской деформации, именно, применения импульсной, линейной нагрузки к одной стороне анизотропюой пластинки. Инверсия для некоторых материалов высодится на основе оси эпицентра. Приводится численный способ решений для обоих случаев.
} 